\title{
General earth fault protection for MV networks using wavelet decomposition and Bayesian criterion
}

\author{
Lubomir Marciniak ${ }^{1, *}$ \\ ${ }^{1}$ Institute of Electric Power Engineering, Technical University of Czestochowa, Aleja Armii \\ Krajowej 17, 42-200 Czestochowa, Poland
}

\begin{abstract}
For selective detection of earth faults in medium voltage networks, the general protection can be used, which unlike individual protections controls earth currents in all lines supplied from the substation. To detect high-resistance and arc faults, it is possible to use in such protections the details from wavelet decomposition of earth currents and the Bayesian conditional probability criterion. The paper presents a protection model using details of earth currents and the Bayesian criterion. Significant parameters of the model were determined: sampling frequency, wavelet types and decomposition levels, parameters of the probability density function and decision conditions. The effectiveness of the protection in the case of non-stationary high-resistance short-circuits in the network with natural asymmetry and harmonic distortion was also assessed.
\end{abstract}

\section{Introduction}

Earth faults in medium voltage networks can be detected by means of individual protections used on each outgoing line or a general protection, which controls earth currents of all outgoing lines in the switchgear [1]. The control of all earth currents provides additional detection possibilities for short circuits compared to individual protections. In practice, general protections are most commonly used, in which the fundamental or higher harmonics all the earth currents are compared [2-4]. The comparison of the fundamental harmonics of earth currents is realized in the protection for the network with an isolated neutral point. That protection detects a short circuit on the line in which the earth current is the highest. In compensated networks, the use of such a criterion is impossible, because as a result of compensation the fundamental harmonic of the earth current is small and often is smaller than own currents of healthy lines, especially long cable lines. In such networks, however, it is possible to identify a faulted line basing on a comparison of mean, effective or amplitude values of higher harmonics that are not compensated. Modern general microprocessor protections enable parallel (simultaneous) measurement and comparison of earth currents, thanks to which they can operate in both steady and transient states.

* Corresponding author: lubmar@el.pcz.czest.pl 
Comparative-current protections have a number of advantages. Unlike individual overcurrent protections, they are easy to use, as they do not require detuning from step changes in the capacitive currents of each outgoing line. Therefore they are much more sensitive and more selective. Compared to directional protections they are much easier to install and support. However, they also have disadvantages. The number of outgoing lines can not be less than three. Due to the limited accuracy of earth current measurements, they are able to distinguish currents with an accuracy of about $10 \%$ of the total network earth current. In addition, such a protection is activated after fulfilling a criterion for a zero sequence voltage, which limits its sensitivity. In practice, protections using the current comparison criterion detect earth faults with a fault resistance $R_{F}$ not exceeding $2 \div 4 \mathrm{k} \Omega$, which is considered insufficient.

Recently many techniques have been proposed to improve the detection of high resistance earth faults in distribution systems. In one of the interesting solutions, it was proposed to use wavelet decomposition for the extraction of high-frequency components (details) of line currents, calculating normalized sums of details and to use Bayesian criterion in relation to these sums in order to detect a short-circuit and to determine selectively a faulted line [5-7].

According to the authors, this method allows to detect earth faults with very high resistance up to $200 \mathrm{k} \Omega$. However, the authors do not specify how to implement the protection, how to choose its parameters or how the technical parameters of the network affect the sensitivity of such a protection. In order to determine the real possibilities of such a protection, its model was built in Matlab/Simulink and subjected to simulation tests.

\section{Protection model}

The general protection model using the Bayesian criterion developed for the purpose of simulation research is shown in Figure 1. This is a hierarchical model consisting of masked multi-channel function blocks. In the presented version, it can supervise six outgoing lines in six channels. Its main blocks are TwoLevelDec realizing two-level wavelet decomposition and adding details of earth currents, Bayes_Fn function block calculating the conditional probability and a decision block DecisionBlock.

The TwoLevelDec block realizes in each channel (Fig. 1b) the initial analog low-pass filtration $(L P F 1)$ of the earth current $I_{E}$, its two-level wavelet decomposition (Decomp 11 , Decomp 12 elements and a downsampling element $D S 1$ ), collects the last samples of current details in a series-parallel register Delay Line 1 and finally calculates the absolute sum of the current detail in the measurement window using the Abs 1 and Sum 1 elements.

Similarly to the classical solutions, the basic quantities for short-circuit detection are the higher harmonics of earth currents $I_{E}$ of all outgoing lines from the MV switchgear section. However, to extract these harmonics, two-level wavelet decomposition is proposed, which is implemented in the TwoLevelDec block. According to own research [8,9], in the case of high-resistance short-circuits in earth currents there are components with relatively small frequencies on the order of $125 \div 250 \mathrm{~Hz}$. Components with higher frequencies are suppressed by fault resistance. In the protections, the sampling frequency $f_{p}=1000 \mathrm{~Hz}$ is most commonly used. At this frequency $f_{p}$, it is necessary to use a two-level wavelet decomposition to detect details from the above-mentioned frequency range. Due to the need to effectively suppress the mains frequency $f_{0}=50 \mathrm{~Hz}$, it be should used wavelet filters with a large number of coefficients. Good results are obtained after applying to the wavelet decomposition of Daubechies filters type $\mathrm{db} 20$ or higher order. In that case, the attenuation of the signal component with the frequency $f_{0}$ is equal $A_{f 0}=103.5 \mathrm{~dB}$. 


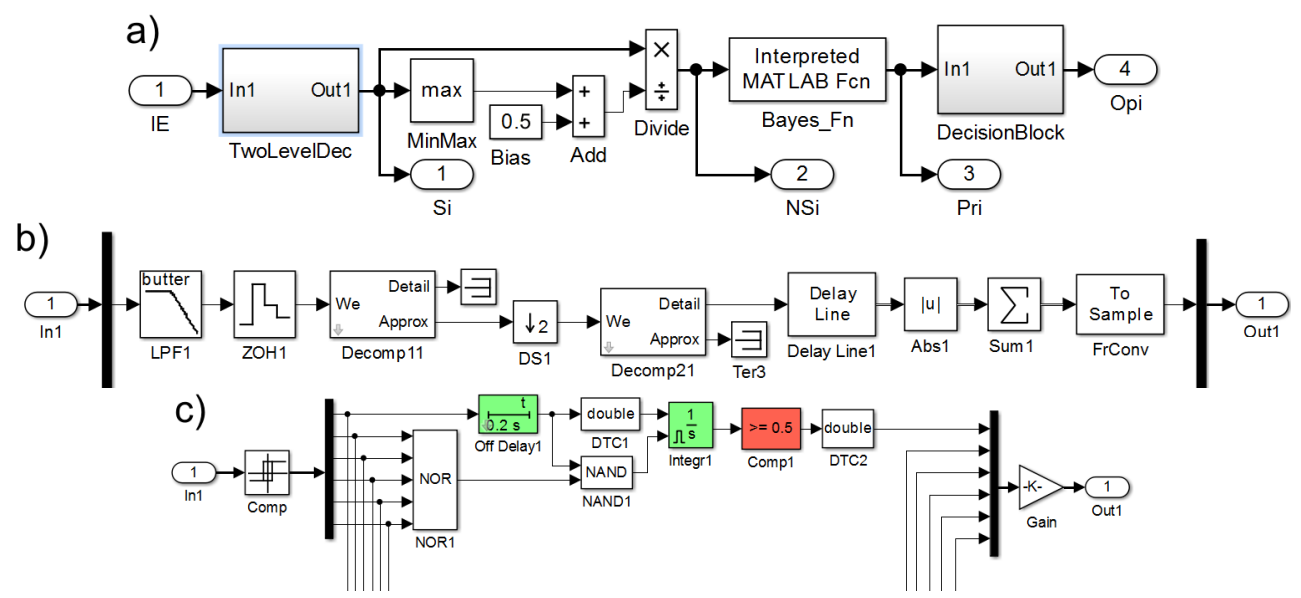

Fig. 1. Model of the general earth fault protection (a), model of one channel of two level wavelet decomposition and sum details (b), model of one channel of logical and decision functions (c).

For all lines, absolute sums of details are created in a measuring window with the length of one cycle of the fundamental harmonic:

$$
S_{i}=\sum_{k=0}^{N-1}\left|d I 2_{k}\right|
$$

where: $N$ - number of samples in the window (window length), $i=1 \div 6$ - numbers of a line (channels of digital signal processing). Window with a length of one cycle of the fundamental harmonic was used so $N=10$ because the details are sampled with a frequency $f_{p} / 2$.

Then, the normalized sums, related to the maximum value, are calculated:

$$
S N_{i}=S_{i} /\left(\max \left(S_{i}\right)+\text { Bias }\right)
$$

where Bias means for a preliminary shift in order to detuning from network disturbances and asymmetry currents.

For signals prepared in this way, the Bayesian conditional probability of short-circuit occurrence in each line is calculated (Bayes_Fn function block):

$$
\operatorname{Pr}\left(i \mid S N_{i}\right)=\frac{f_{1}\left(N S_{i}\right)}{f_{0}\left(N S_{i}\right) \sum_{i=1}^{K} f_{1}\left(N S_{i}\right) / f_{0}\left(N S_{i}\right)}
$$

In equation (3), the functions $f_{1}\left(S N_{i}\right)$ and $f_{0}\left(S N_{i}\right)$ denote, respectively, the probability density of the short-circuit and the lack of short-circuit in the line $i$. They are calculated from dependencies:

$$
\begin{aligned}
& f_{1}\left(N S_{i}\right)=\frac{1}{\sigma_{1} \sqrt{2 \pi}} e^{-\frac{\left(S N_{i}-\mu_{1}\right)^{2}}{2 \sigma_{1}^{2}}} \\
& f_{0}\left(N S_{i}\right)=\frac{1}{\sigma_{0} \sqrt{2 \pi}} e^{-\frac{\left(S N_{i}-\mu_{0}\right)^{2}}{2 \sigma_{0}^{2}}}
\end{aligned}
$$


where: $\sigma_{0}, \sigma_{1}$-standard deviations, $\mu_{0}, \mu_{1}$ - expected values.

As a result of own research, it was found that optimal values of standard deviation should be $\sigma_{0}=\sigma_{1}=0.2$, and expected values $\mu_{0}=0.2, \mu_{1}=0.8$, respectively. The shape of these functions with such parameters is shown in Figure 2.

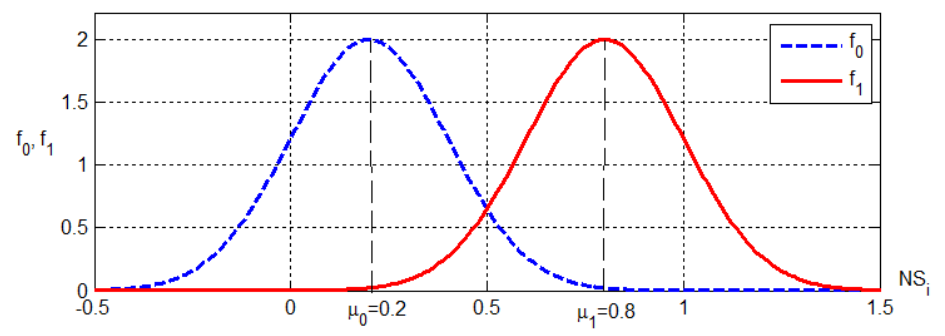

Fig. 2. Probability density functions.

The probability signals are then processed in a DecisionBlock (Fig. 1c). In this block, first of all, the comparison (Comp element) of the signals $\operatorname{Pr}\left(i \mid S N_{i}\right)$ with the threshold value $P r_{r e f}$ is made:

$$
C o_{i}=\left(\operatorname{Pr}_{i}\left(i \mid N S_{i}\right)>P r_{r e f}\right)
$$

In the case of the above-mentioned parameters of the probability functions, $P r_{r e f} \geq 0.5$ should be used.

A high level on the comparator output means a high probability of a short circuit. However, these signals are not enough to identify a faulty line for several reasons. Firstly, they may be discontinuous (in the form of short impulses) in the case of intermittent arc faults. Secondly, the high state can occur simultaneously at the output of several comparators during switching operations in the network or lightning discharges. Thirdly, during short-circuits in a mixed (overhead-cable) network with a high harmonics content in the supply voltage, the criterion (6) can be met for several lines (uncertainty area), especially for long cable lines, which leads to erroneous identification of the short-circuit.

To detect intermittent faults, a logical inertia was applied, consisting in the elongation of output signals from comparators. This function was performed using the Off Delay 1 block (Fig. 1c). The $O D 1$ signal at the output of this block becomes continuous if the time interval between consecutive impulses from the comparator $C o 1$ does not exceed the $T_{O D}$ interval. To detect intermittent short-circuits occurring sporadically, $T_{O D} \geq 0.2 \mathrm{~s}$ should be used. To exclude erroneous operations of the protection under the influence of switching disturbances, the time delay $T_{d e l}$ (elements Integr 1 and Comp 1 in Fig. 1c) was used. This delay should be at least 2.5 times greater than the $T_{O D}$. In practice, this means that $T_{\text {del }} \geq 0.5 \mathrm{~s}$.

To exclude erroneous actions of the protection in the event of activation of several comparators (earth fault on the cable line), logical functions were used to reset the delay elements in each channel if there is an activation in any other channel. This logic is implemented by the NOR 1 and NAND1 blocks. Reset of the delay element in channel 1 $\left(R e_{d e l 1}=0\right.$ signal $)$ will occur after the following logical dependence has been met:

$$
R e_{d e l 1}=\overline{\overline{C o_{2} \cup C o_{2} \cup \ldots \cup C o_{6}} \cap O D_{1}}
$$

where: $\underline{O D_{1}}$ - output signal from an Off Delayl block.

Similar logical relationships are realized in other protection channels. Reset signals $R e_{\text {del } i}$ prevent erroneous operations of the protection in the uncertainty area. 


\section{Tests of the protection}

The presented protection model was subjected to intensive tests to explain its effectiveness. The model of the compensated $15 \mathrm{kV}$ network built from four overhead lines and two cable lines with a total capacitive current $I_{C S}=46.8 \mathrm{~A}$ was used for the tests (Fig. 3). Table 1 lists the lengths of individual lines $l_{i}$ and the share coefficients of these lines in the network capacitance $\alpha_{i}$. The network model takes into account the natural capacitive asymmetry of overhead lines, which is $2.5 \%$. The harmonic distortion of supply voltages, mainly the 3rd harmonic, has also been taken into account in order to explain its influence on the operation of the protection. The maximum permissible content of 3rd harmonic equal to 5\% [10] has been assumed. The non-linear earth-fault resistance $R_{F}$ was modeled as a series circuit consisting of the arc resistance $R_{\text {arc }}$ [11-14] and the transition resistance $R_{T}$. The arc model has been used with parameters $[11,12]$ : ignition voltage $U_{i g}=10 \mathrm{kV}$, voltage in the arc column in a conducting state $U_{k}=1000 \mathrm{~V}$, resistance in a conducting state $R_{1}=0.1 \Omega$, time constant $\tau=0.1 \mathrm{~ms}$. The protection was tested both in the case of low-resistance and highresistance short-circuits located at various points of the network marked in Figure 3. For this purpose, the value of the transition resistance $R_{T}$ in a wide range was changed.

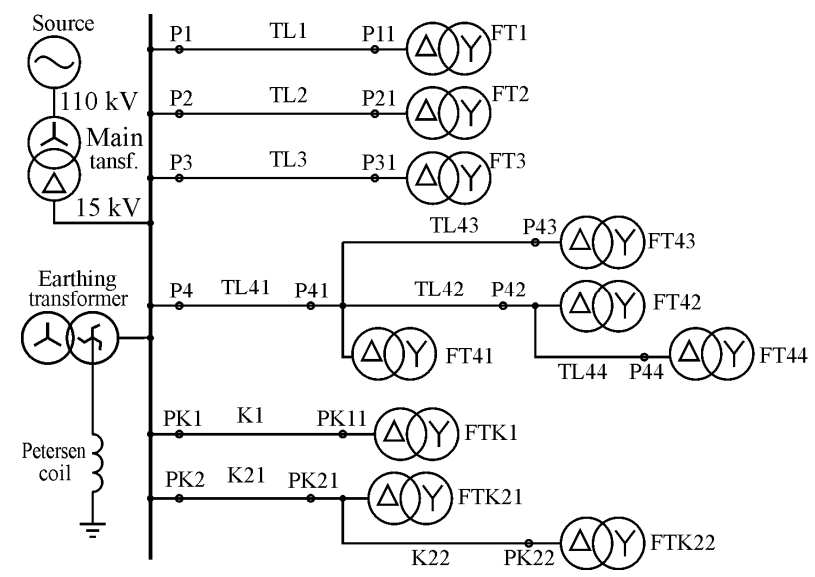

Fig. 3. General diagram of the modelled MV network.

Table 1. Line lengths $l$ and line share coefficients in the network capacitance $\alpha$.

\begin{tabular}{|c|c|c|c|c|c|c|}
\hline Line & TL1 & TL2 & TL3 & TL4 & K1 & K2 \\
\hline$l,[\mathrm{~km}]$ & 30 & 50 & 70 & 53 & 15 & 17 \\
\hline$\alpha$ & 0.023 & 0.038 & 0.054 & 0.041 & 0.396 & 0.449 \\
\hline
\end{tabular}

At the beginning, a very important Bias parameter was defined. Its value was estimated from the condition of non-operation of the protection in the worst working conditions of the network without short-circuit: natural asymmetry of shunt parameters, maximum content of the third harmonic in supply voltages $k_{3}=5 \%$, exact compensation $(s=0)$. The value of Bias $=0.5$ was assumed. This is many times more than suggested in [7]. Figure 4 presents the asymmetry currents $I_{E}$, normalized sums of details $N S$ and conditional probabilities $\mathrm{Pr}$ in the described case. The largest value is assumed by the signal $\operatorname{Pr}_{6}$ for cable line K2 (6) and it is $20 \%$ smaller than the comparator threshold $\operatorname{Pr}_{\text {ref }}=0.5$. 


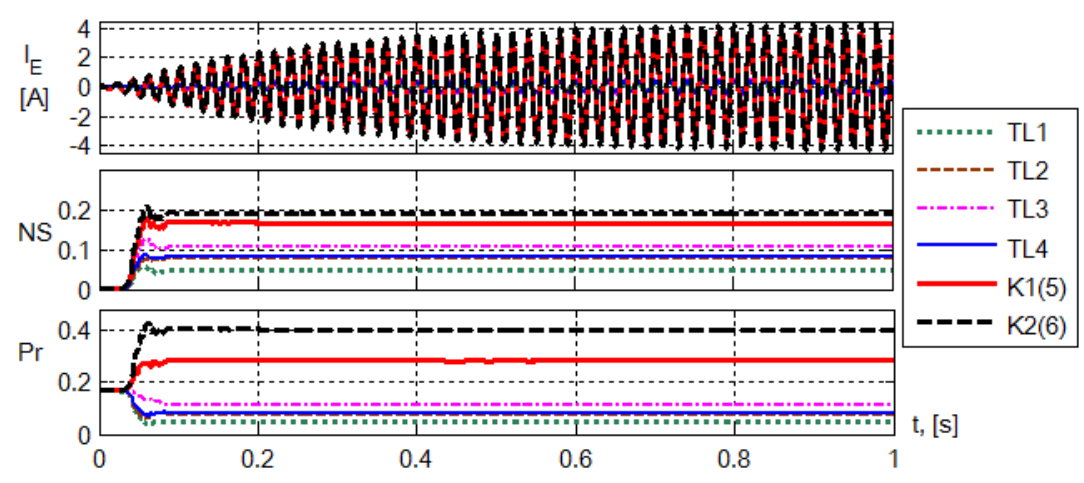

Fig. 4. Asymmetry earth currents $I_{E}$, normalized sums of details $N S$ and conditional probabilities $P r$ in the worst working conditions of the network: natural asymmetry, $k_{3}=5 \%, \mathrm{~s}=0$, Bias $=0.5$.

Detailed simulation tests have shown that the protection selectively detects short circuits, both low-resistance and high-resistance, in overhead lines TL $1 \div$ TL4 regardless of their location on the line (distance from the substation). Figure 5 shows an example of the fault detection at a point P41 ( $l=10 \mathrm{~km}$ from the substation) of the overhead line TL4, in the case of a low-resistance and high-resistance short-circuit. In both cases, only one signal $P r_{4}>P r_{\text {ref }}=0.5$ and a short circuits are detected on the line TL4 (the logical signal $O p_{4}$ takes the value 1 when $t \geq 0.69 \mathrm{~s})$. The examples of short-circuit detection presented in Figure 5 were obtained in the compensated network with detuning $s=0.1$ and a high share of the third harmonic in the supply voltage $k_{3}=5 \%$.
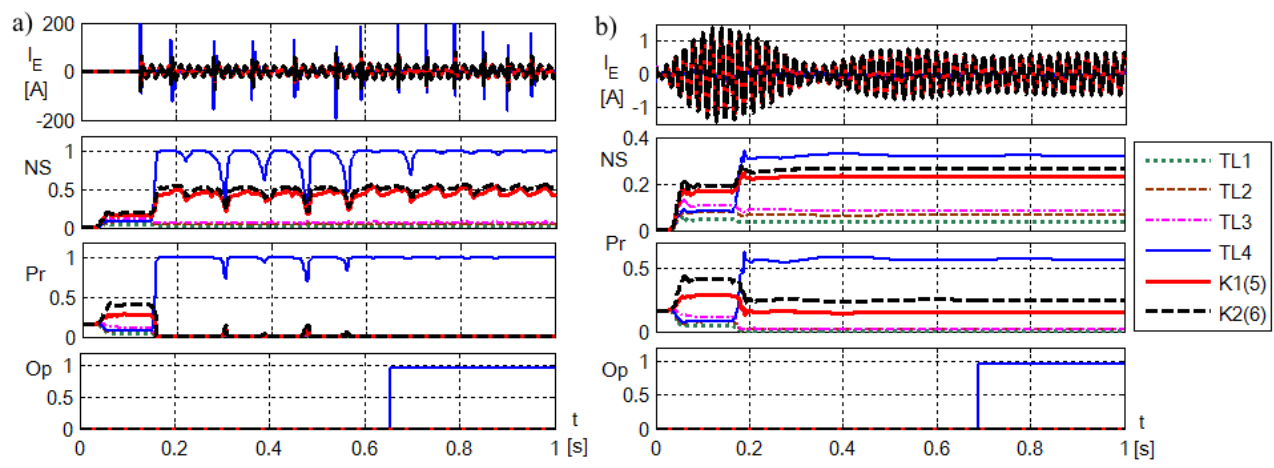

Fig. 5. Signals in the protection during short-circuit at a point P41 of the line TL4: a) $R_{T}=1 \Omega$, b) $R_{T}=80 \mathrm{k} \Omega$.

Correct detection of short circuits on cable lines $\mathrm{K} 1$ and $\mathrm{K} 2$ is possible only in noncompensated networks $(s=-1)$.

In a compensated network with a high content of the third harmonic $\left(k_{3}=5 \%\right)$, the protection does not detect a low-resistance short-circuit in cable lines, and in case of a highresistance short-circuit it detects short-circuit incorrectly.

Such cases are shown in Fig. 6. During low-resistance short-circuit at point PK11 (Fig. 6a), signals $\mathrm{Pr}_{5}$ and $P r_{6}$ corresponding to lines $\mathrm{K} 1$ and $\mathrm{K} 2$ exceed the threshold value $P r_{r e f}$ and the logic (Fig. 1c) blocks the operation of the protection. During high-resistance short-circuit at a point PK11 (Fig. 6b), the normalized sum of details of the line K2 is greater than the sum of the line $\mathrm{K} 1\left(N S_{6}>N S_{5}\right)$ and therefore there is an erroneous fault detection in the line $\mathrm{K} 2$ instead of $\mathrm{K} 1\left(\mathrm{Pr}_{6}>\mathrm{Pr}_{\text {ref }}\right.$ and $\left.O p_{6}=1\right)$. The protection also does not detect low-resistance faults at cable lines in a compensated network even in case the 
supply voltage does not contain harmonics in the frequency range of $124-250 \mathrm{~Hz}$, including 3rd harmonic $\left(k_{3}=0\right)$.
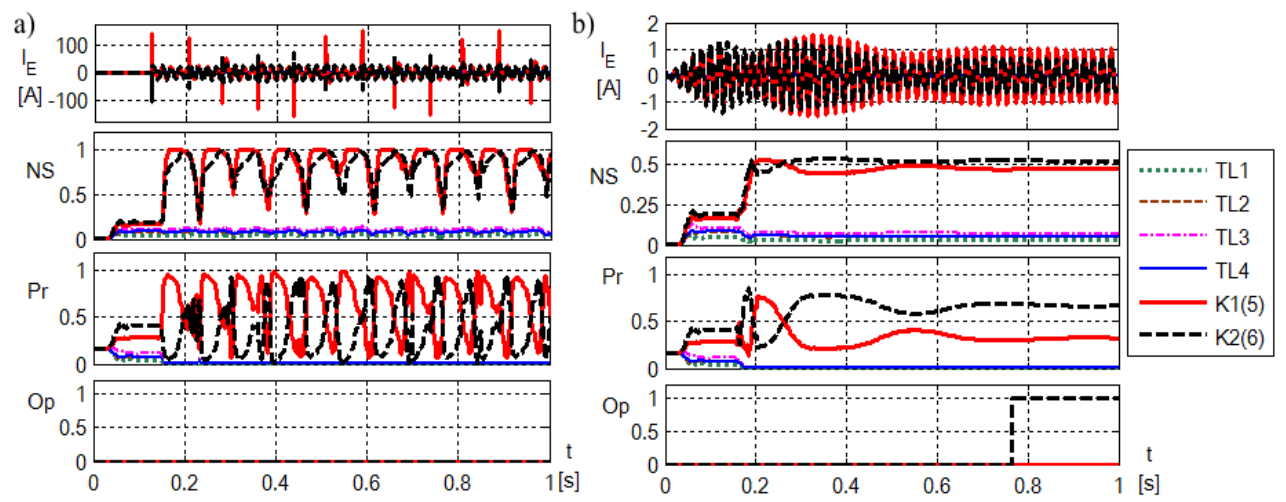

Fig. 6. Signals in the protection during short-circuit at a point PK11 of the line K1: a) $R_{T}=10 \Omega$, b) $R_{T}=20 \mathrm{k} \Omega$.

Table 2 presents the maximum values of the transition resistance $R_{T}$, at which protection operates during faults at different points of the network. They were determined by simulations for the network with an isolated neutral point $(s=-1)$, an undercompensated network $(s=-0.1)$ and in an overcompensated network $(s=0.1)$ with the high third harmonic content $k_{3}=5 \%$ in the supply voltage. The maximum $R_{T}$ values in the compensated network without harmonics in the supply voltage were also determined $\left(k_{3}=0 \%\right)$.

The presented results show very high effectiveness in detection high-resistance earth faults in overhead lines. A relatively small influence on this effectiveness has a compensation detuning and significant influence has a harmonic content in the supply voltage. Earth faults with the highest resistance $R_{T}$ are detected in the network with isolated neutral point. Compensation causes a slight reduction in the components of the current in the frequency range $125 \div 250 \mathrm{~Hz}$ and as a result short-circuits with a smaller $R_{T}$ value are detected. The detailed dependence of $R_{T}$ on detuning $s$ for a point P41 is presented in Table 3. In the compensated network without the participation of higher harmonics in the supply voltage, the maximum transition resistance is usually more than twice as high as in the network with $k_{3}=5 \%$. Detection of earth faults in cable lines with a large share of line capacitance $(\alpha>0.4)$ is not possible. Therefore, the protection in cable lines should be blocked.

The described protection is sensitive to the network switching operations. Figure 7 shows an example of the protection's response to the switching off $(t=0.35 \mathrm{~s})$ and switching on operations $(t=0.5 \mathrm{~s})$ of the line segment TL43 together with the transformer FT43. After switching off the comparator $\mathrm{Co}_{4}$ operates because $\mathrm{Pr}_{4}>\mathrm{Pr}_{\text {ref }}$, and after switching on - the comparator $\mathrm{Co}_{6}$ operates because $\mathrm{Pr}_{6}>\mathrm{Pr}_{\text {ref. }}$. However, the protection does not operate, because for such a circumstance a logical lock and a time delay were used. The logical lock works in the event of activation in several channels at intervals no greater than $T_{O D}$, while the time delay locks the operation of the protection under the influence of single disturbances. 
Table 2. The maximum resistance of the earth faults at which the protection operates.

\begin{tabular}{|c|c|c|c|c|c|}
\hline$><$ & 3rd harmonic $k_{3},[\%]$ & \multicolumn{3}{|c|}{5} & 0 \\
\hline$>$ & Detuning $S$ & -1.0 & -0.1 & 0.1 & 0.1 \\
\hline Point & Distance $l,[\mathrm{~km}]$ & \multicolumn{4}{|c|}{$R_{T},[\mathrm{k} \Omega]$} \\
\hline P1 & 0 & 97 & 89 & 87 & 227 \\
\hline P12 & 30 & 121 & 106 & 94 & 252 \\
\hline P2 & 0 & 113 & 102 & 88 & 219 \\
\hline P21 & 50 & 142 & 123 & 107 & 248 \\
\hline P3 & 0 & 131 & 114 & 92 & 213 \\
\hline P31 & 70 & 140 & 111 & 115 & 218 \\
\hline P4 & 0 & 115 & 103 & 88 & 218 \\
\hline P41 & 10 & 128 & 114 & 93 & 234 \\
\hline P42 & 25 & 133 & 118 & 97 & 239 \\
\hline $\mathrm{P} 43$ & 30 & 135 & 119 & 99 & 240 \\
\hline P44 & 33 & 135 & 119 & 99 & 240 \\
\hline Pk1 & 0 & 72 & $*$ & $*$ & $82 * *$ \\
\hline Pk12 & 15 & 58 & * & $*$ & $90^{* *}$ \\
\hline $\mathrm{Pk} 2$ & 0 & 74 & * & $*$ & $80^{* *}$ \\
\hline Pk21 & 10 & 57 & * & $*$ & $84 * *$ \\
\hline Pk22 & 17 & 49 & $*$ & $*$ & $84 * *$ \\
\hline
\end{tabular}

* - non-action in the case of earth faults with resistance $R_{T}<1.7 \mathrm{k} \Omega$ due to excitation in cable lines $\mathrm{K} 1$ and $\mathrm{K} 2$ and non-selective operation at high transition resistances.

** - non-action in the case of short-circuits with resistance $R_{T}<1.7 \mathrm{k} \Omega$ due to excitation in cable lines $\mathrm{K} 1$ and $\mathrm{K} 2$ and blocking.

Table 3. The maximum transition resistance depending on the compensation detuning for the earth fault at P41

\begin{tabular}{|c|c|c|c|c|c|c|c|c|c|c|c|c|c|c|}
\hline$S$ & -1 & -0.7 & -0.5 & -0.3 & -0.2 & -0.15 & -0.1 & -0.05 & 0 & 0.05 & 0.1 & 0.15 & 0.2 & 0.3 \\
\hline$R_{T},[\mathrm{k} \Omega]$ & 128 & 121 & 117 & 114 & 113 & 114 & 114 & 119 & 92 & 88 & 93 & 94 & 95 & 92 \\
\hline
\end{tabular}

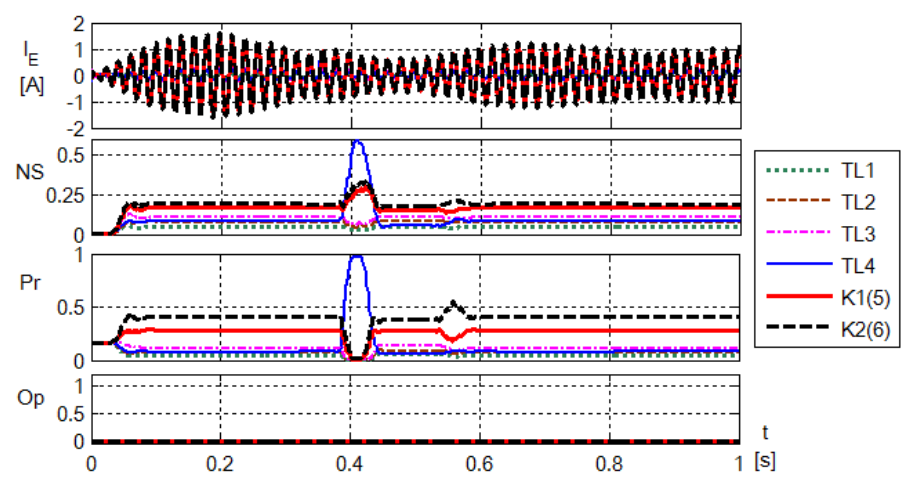

Fig. 7. The response of the protection to the switching off $(t=0.35 \mathrm{~s})$ and switching on $(t=0.5 \mathrm{~s})$ of the line segment TL43.

\section{Conclusions}

Earth faults in medium voltage networks can be detected by means of general protection, controlling earth currents of all outgoing lines in the switchgear. In one of the new methods of detection of high-resistance earth faults in compensated MV networks, it was proposed to use wavelet decomposition to extract high-frequency components (details) of the line 
currents, calculate normalized sums of details and Bayesian criterion for these sums. According to the authors, this method allows to detect of earth faults with very high resistance up to $200 \mathrm{k} \Omega$.

In order to determine the real possibilities of such a protection, its model was built in Matlab/Simulink and subjected to simulation tests using the model of $15 \mathrm{kV}$ compensated network built of four overhead lines and two cable lines. The protection model uses details from two-level wavelet decomposition in the frequency range 125-250 Hz. The network model includes the natural capacitive asymmetry of the overhead lines and harmonic distortion, mainly the $3 \mathrm{rd}$ harmonic, in the supply voltages. Complex earth faults have been modeled with the use of dynamic arc resistance and transition resistance.

Detailed simulation tests have shown that the protection detects earth faults selectively in the network with harmonic distortions, both low-resistance and high-resistance only in overhead lines. Short-circuits with a transition resistance from 87 to $142 \mathrm{k} \Omega$ are detected, depending on the fault location and compensation detuning. In a network without harmonic distortion in the supply voltages, short-circuits with resistance of about twice as high (up to $252 \mathrm{k} \Omega$ ) as in the network with harmonic distortions are detected.

Detection of earth faults in cable lines with a large share in the capacitance $(\alpha>0.4)$ is only possible in the network with isolated neutral point. Earth faults on cable lines in compensated networks are not detected $\left(R_{T}<1.7 \mathrm{k} \Omega\right)$ or they are detected incorrectly $\left(R_{T}>1.7 \mathrm{k} \Omega\right)$. Therefore, the operating of the protection in cable lines with a large capacitance should be blocked. Detection of high-resistance short-circuits on all lines of the compensated network is only possible with similar capacitances of the lines.

To detect intermittent arc faults, a logical inertia with a time delay of $T_{O D} \geq 0.2 \mathrm{~s}$ should be used in the protection. The protection should work with a time delay not lower than $T_{d e l} \geq 2.5 T_{O D}=0.5 \mathrm{~s}$ in order to prevent erroneous operations due to switching disturbances.

\section{References}

1. S. Hänninen, Single phase earth faults in high impedance grounded networks. Characteristics, indication and location, Technical Research Centre of Finland, Espoo 2001

2. F. Rodoń, R. Głowocz, M. Talaga, Microprocessor ground fault protection type ZIo. Scientific papers of the Institute of Electric Power Engineering of the Technical University of Wrocław, 86, 75-86, (1997) (in Polish)

3. V.F. Bukhtoyarov, Adaptive current protection from ground short-circuits in electric installations 6-10 kV and the experience of its application, South Ural State University Bulletin, 16, 70-75, (2012) (in Russian)

4. MKZZP-6-35-К. Microcontroller device for protecting of the busbar section feeders from single-phase-to-earth short circuits in 6-35 $\mathrm{kV}$ compensated networks. User manual, Novosibirsk, (2009) (in Russian)

5. N.I. Elkalashy, M. Lehtonen, N.G. Tarhuni, DWT and Bayesian technique for enhancing earth fault protection in MV network, 2009 IEEE/PES Power Systems Conference and Exposition, 15-18 March, (2009)

6. N.I. Elkalashy, A.M. Elhaffar, T.A. Kawady, N.G. Tarhuni, M. Lehtonen, Bayesian selectivity technique for earth fault protection in medium-voltage networks. IEEE Transactions on Power Delivery, 25/4, 2234-2245, (2010) 
7. N.I. Elkalashy, M. Lehtonen, Advancing DWT-Bayesian technique for earth fault protection in $M V$ networks, The Online Journal on Electronics and Electrical Engineering (OJEEE), 1/2, 89-93, (2009)

8. L. Marciniak, Impulse-current and wavelet criteria of resistive and arc earth fault identification in the medium voltage networks, Wydawnictwo Politechniki Częstochowskiej, Monographs No 273, (2013) (in Polish)

9. L. Marciniak, Wavelet criteria for identification of arc intermittent faults in medium voltage networks, Proceedings of the International Symposium "Modern Electric Power Systems MEPS'10", paper 09.1, Wrocław, 20-22 September, (2010)

10. PN-EN 50160:2010P. Voltage characteristics of electricity supplied by public electricity networks

11. L. Marciniak, Model of the arc earth-fault for medium voltage networks, Central European Journal of Engineering, 1/2, 168-173, (2011)

12. L. Marciniak, Implementations of the arc earth faults model in programs PSCAD and Matlab/Simulink, Przegląd Elektrotechniczny, 88/9a, 126-129, (2012) (in Polish)

13. A. Sawicki, M. Haltof, Nonlinear mathematical models of the electric arc, Przegląd Elektrotechniczny, 92/11, 257-261, (2016) (in Polish)

14. A. Sawicki, Rationalizations of hybrid electrical arc models, Przegląd Elektrotechniczny, 93/11, 198-203, (2017) (in Polish) 